\title{
Serum levels of platelet released CD40 ligand are increased in early onset occlusive carotid artery disease
}

\author{
József Balla ${ }^{\mathrm{a}, 1}$, Mária Tünde Magyar ${ }^{\mathrm{b}, 1, *}$, Dániel Bereczki ${ }^{\mathrm{b}}$, Attila Valikovics $^{\mathrm{c}}$, Emöke Nagy ${ }^{\mathrm{a}}$, \\ Erika Barna ${ }^{\mathrm{a}}$, András Pál ${ }^{\mathrm{d}}$, György Balla ${ }^{\mathrm{e}}$, László Csiba ${ }^{\mathrm{b}}$ and György Blaskó ${ }^{\mathrm{d}}$ \\ ${ }^{a}$ Department of Medicine, University of Debrecen, Medical and Health Science Center, Debrecen, Hungary \\ ${ }^{\mathrm{b}}$ Department of Neurology, University of Debrecen, Medical and Health Science Center, Debrecen, Hungary \\ ${ }^{\mathrm{c}}$ Department of Neurology, County Hospital, Miskolc, Hungary \\ ${ }^{\mathrm{d}}$ Sanofi-Synthelabo Hungary Ltd., Medical Department, Budapest, Hungary \\ ${ }^{\mathrm{e}}$ Department of Neonatology, University of Debrecen, Medical and Health Science Center, Debrecen, Hungary
}

\begin{abstract}
Objective: Soluble CD40 ligand (sCD40L) has been suggested as a key mediator between inflammation and atherosclerosis, and the CD40-CD40L interaction has a role in atherosclerotic lesion progression. We evaluated if platelet released serum sCD40L and SCD40 levels differ between patients with early onset occlusive carotid artery disease and age-matched controls. Methods: sCD40L and sCD40 levels were measured in serum samples of 60 patients with occlusive carotid artery disease and 30 age-matched controls using ELISA. Degree of stenosis of the internal carotid artery (ICA), and intima-media thickness (IMT) in the common carotid artery were measured by high resolution ultrasound. Values are given as mean $\pm \mathrm{SD}$.

Results: Mean age was $50.9 \pm 3.5$ and $50.1 \pm 3.5$ years in the patient and control groups. IMT was significantly thicker in patients than in controls $(0.89 \pm 0.14$ vs. $0.78 \pm 0.12 \mathrm{~mm}, p=0.0003)$. Serum levels of sCD40L were significantly higher (6.9 \pm 5 vs. $4.5 \pm 3.0 \mathrm{ng} / \mathrm{mL}, p=0.038)$ in patients, whereas sCD40 did not differ significantly between patients and controls ( $85 \pm$ 56.9 vs. $79.3 \pm 18.7 \mathrm{pg} / \mathrm{mL}, p=0.34)$. IMT did not correlate with sCD40L or sCD40 levels $(R=-0.03, p=0.77$; and $R=$ $0.109, p=0.308$, respectively).

Conclusions: sCD40L but not sCD40 levels are significantly higher in patients with occlusive carotid artery disease. Platelet derived SCD40L may be a key mediator among inflammation, thrombosis and atherosclerosis.
\end{abstract}

Keywords: CD40 ligand, CD40, atherosclerosis, inflammation, intima-media thickness

\section{Introduction}

CD40 ligand (CD40L), a trimeric transmembrane protein of the tumor necrosis factor (TNF) family, was originally identified on the surface of activated CD4+ helper T cells [1]. In recent years, CD40L has emerged

\footnotetext{
${ }^{1}$ The first two authors contributed equally to these studies.

* Corresponding author: Mária Tünde Magyar, M.D., Ph.D., Department of Neurology, Medical and Health Science Center, University of Debrecen, Nagyerdei krt. 98., Debrecen, H-4012, Hungary. Tel.: +36 52415 176; Fax: +36 52453 590; E-mail: tundus@ jaguar.unideb.hu.
}

as a pleiotropic mediator of immune and inflammatory processes [2]. Out of these are the induction of adhesion molecule expression on endothelial cells (e.g. Eselectin, VCAM-1) and platelet activation causing local inflammation of the vessel wall which consequently leads to atherosclerosis [3,4]. Recent work has demonstrated that the CD40L-CD40 signaling is critical in the pathogenesis of atherosclerosis [5].

Henn and coworkers [6] showed that CD40L and its receptor CD40 exist in platelets. CD40L is cryptic in unstimulated platelets but rapidly presented to the surface after stimulation. The surface-expressed CD40L is then cleaved from the platelet subsequently generating 
a soluble fragment (soluble CD40 ligand or sCD40L). Both membrane-associated and sCD40L may induce procoagulant activity [7]. It is estimated that most of the circulating SCD40L is derived from platelets [8].

Recent studies found increased levels of sCD40L in patients with acute coronary syndromes [9], with acute cerebral ischemia [10] and with peripheral arterial occlusive disease [11]. Moreover, upregulation of the CD40/CD40L dyad was found in cardiovascular risk factors, in diabetes mellitus [12], in hypercholesterolemia [13] and in smoking status [14]. Elevated levels of SCD40L were found to serve as a risk factor for future cardiovascular events in healthy women [15].

In our previous studies we found higher white blood cell count (WBC), C-reactive protein (CRP) and fibrinogen levels in patients with early onset occlusive carotid artery disease suggesting the role of inflammation [16]. In the present study we evaluated whether serum sCD40L and sCD40 levels differ between patients with occlusive carotid artery disease and agematched controls. Age is the primary risk factor for carotid artery disease [17] and examining an elderly patient group may mask the effects of risk factors for early atherosclerosis. Therefore for the present study we selected subjects with occlusive carotid artery disease below 60 years of age.

Intima-media thickness (IMT) measured by B-mode ultrasound in the common carotid arteries (CCA) is a sensitive marker of atherosclerosis [18] and prospective studies have shown that increased IMT of the CCA is a powerful predictor of coronary and cerebrovascular complications [19]. IMT was linearly related to the total number of cardiovascular risk factors [20] and increased CCA IMT was found with elevated CRP levels [21]. For these reasons we evaluated the relationship between the sCD40L level and CCA IMT measured by B-mode Doppler in young and middle aged subjects.

\section{Methods}

\subsection{Patient population}

Patients were recruited at the Neurosonological Laboratory of the Department of Neurology, University of Debrecen. Patients below 60 years of age and with an at least $30 \%$ internal carotid artery (ICA) stenosis or occlusion at screening were invited to participate in the study. An age- and gender matched control group was recruited who had no plaques or stenosis of the carotid arteries by duplex examination. Most of these controls were treated primarily for tension-type headache or anxiety disorder. Subjects with infections, malignancies and autoimmune diseases were excluded. Further exclusion criteria were surgery and stroke within the previous 6 months. After getting information about the purpose of the study the participants signed a consent form before blood sampling and ultrasound examination. The study was approved by the Ethical Committee of the University of Debrecen.

\subsection{Analysis of blood samples}

After completing a risk factor questionnaire fasting blood samples were taken between 7.30 and 8.00 in the morning. Blood samples were kept at room temperature, while samples for homocysteine evaluation were kept on ice and analysis started no later than 90 minutes after blood sampling. Analysis of blood samples was performed by standard laboratory techniques. Homocysteine was determined by the Abbott Axsym system (Abbott Laboratories, Abbott Park, IL, USA) using fluorescence polarization immunoassay. Serum lipids were measured on the Cobas Integra 700 Analyser (Roche, Basel, Switzerland). For measuring of serum sCD40L and sCD40 levels blood was allowed to clot for 90-120 minutes at room temperature before centrifugation for 20 minutes at $4{ }^{\circ} \mathrm{C}$ at $3000 \mathrm{rpm}$. The supernatant was stored at $-80^{\circ} \mathrm{C}$ until analysis. Serum sCD40L and sCD40 levels were measured using ELISA kits of Bender MedSystems (Bender MedSystems, Vienna, Austria). The detection limits of sCD40 and SCD40L defined as the analyte concentration resulting in an absorption significantly higher than that of the dilution medium (mean+3SD) were $12 \mathrm{pg} / \mathrm{mL}$ and $0.095 \mathrm{ng} / \mathrm{mL}$, respectively. Duplicate samples were measured and the mean of the two values was used for further calculation.

\subsection{Carotid duplex ultrasound investigations}

Ultrasound examinations were performed immediately after blood sampling using a color-coded HP SONOS 4500 (Hewlett Packard, Andover, Mass., USA) carotid duplex equipment with a $7.5 \mathrm{MHz}$ linear transducer. Both diameter and area reductions were measured according to the European Carotid Surgery Trial (ECST) method [22]. For screening, the ICA stenosis was classified in categories of $10 \%$ taking into account the peak systolic velocity in the jet of the stenosis, the broadening of the stenotic and poststenotic spec- 
Table 1

Demographic characteristics and risk factor distribution of study participants

\begin{tabular}{lccc}
\hline Feature & Controls & Patients & $\mathrm{P}$ \\
\hline $\mathrm{N}$ & 30 & 60 & \\
Male/female & $15 / 15$ & $36 / 24$ & 0.3668 \\
Age (years) (mean $\pm \mathrm{SD})$ & $50.2 \pm 3.5$ & $51 \pm 3.5$ & 0.423 \\
Hypertension (Y/N) & $12 / 18$ & $43 / 17$ & 0.0037 \\
Diabetes (Y/N) & $1 / 29$ & $5 / 55$ & 0.3436 \\
Smoking (Y/N) & $6 / 24$ & $36 / 24$ & 0.0003 \\
Hyperlipidemia (Y/N) & $9 / 21$ & $31 / 29$ & 0.0512 \\
Heart disease (Y/N) & $6 / 24$ & $18 / 42$ & 0.3119 \\
Ischemic stroke or TIA in history (Y/N) & $2 / 28$ & $29 / 31$ & $<0.0001$ \\
Stroke in family history (Y/N) & $13 / 17$ & $22 / 38$ & 0.5408 \\
Antiplatelet treatment (Y/N) & $5 / 25$ & $40 / 20$ & $<0.0001$ \\
\hline
\end{tabular}

$\mathrm{P}$ denotes statistical significance of Mann-Whitney $\mathrm{U}$ test for age, and Pearson chi-square test for frequencies.

trum, the peak systolic velocity in the poststenotic ICA, and the direction of ophthalmic flow. Occlusion was diagnosed in the complete absence of detectable flow in and above the stenosis and the presence of corresponding indirect hemodynamic criteria [23].

The ultrasound examinations were videotaped. Offline analysis of CCA IMT was made by an independent reader, blinded to the characteristics of study subjects, on video images based on the Atherosclerosis Risk in Communities (ARIC) study protocol [24]. The IMT was measured in the far wall of the right and left CCA-s on the $1-\mathrm{cm}$ segment proximal to the dilatation of the carotid bulb. In each of this 1-cm segment, 11 measurements of IMT were performed at 1-mm increment. The mean IMT of the 22 values in each patient was calculated.

\subsection{Statistical methods}

Normality of continuous variables was checked by the Shapiro-Wilk test. The Mann-Whitney U-test, the Kruskal-Wallis ANOVA and Spearman correlation were used. Frequencies were compared by the Pearson chi-square test. As both continuous and categorical predictors of sCD40L, sCD40 and IMT were assumed; a general linear model was used to identify correlates of sCD40L or IMT values. To build the model, first univariate Spearman correlations were calculated for sCD40L, sCD40 or IMT. Those continuous variables that were found to correlate with SCD40L or IMT values were entered into the general regression model as continuous variables. Categorical predictors that were found to be significant predictors of SCD40L or IMT values in univariate analyses by the Mann-Whitney test (smoking or hypertensive status) were entered in the final model. Two-sided p-values were used. Statistical significance was assumed when $p<0.05$. Statistica for Windows v.6.1 (StatSoft, Tulsa, OK) was used for data analysis.

\section{Results}

\subsection{Characteristics of study groups}

Sixty patients (16 with internal carotid artery occlusion and 44 with $>30 \%$ carotid artery stenosis) and 30 age-matched controls were investigated. The characteristics of the patient and control groups are given in Table 1. The mean age in the total study population was $50.7 \pm 3.5$ years (mean $\pm \mathrm{SD}$, range $42-58$ years) with no difference between the groups $(p=0.423)$. Gender distribution was similar in the control and patient groups ( $p=0.3668)$. There was no difference in the presence of diabetes $(p=0.3434)$, hyperlipidemia $(p=0.0512)$, heart diseases $(p=0.3119)$, and the family history of cerebrovascular diseases $(p=0.5408)$ between the patient group and controls. Hypertension was more prevalent in patients with occlusive carotid artery disease than in the controls $(p=0.0037)$. More of the patients were smokers $(p=0.0003)$. Ischemic stroke or TIA was more prevalent in patients than in controls $(p<0.0001)$. In the patient group 40 subjects were treated with antiplatelet agents (36 took aspirin, 2 clopidogrel and 2 ticlopidin) while in control group only 5 subjects received antiplatelet treatment (2 aspirin, 1 clopidogrel and 2 ticlopidin $)(p<0.0001)$.

\subsection{Time-dependent release of CD4OL and CD4O}

We measured the time-dependent release of CD40L and CD40 on 6 healthy subjects' samples at room temperature. Soluble CD40L was close to zero in the serum in the first 15 minutes after blood sampling, then the re- 


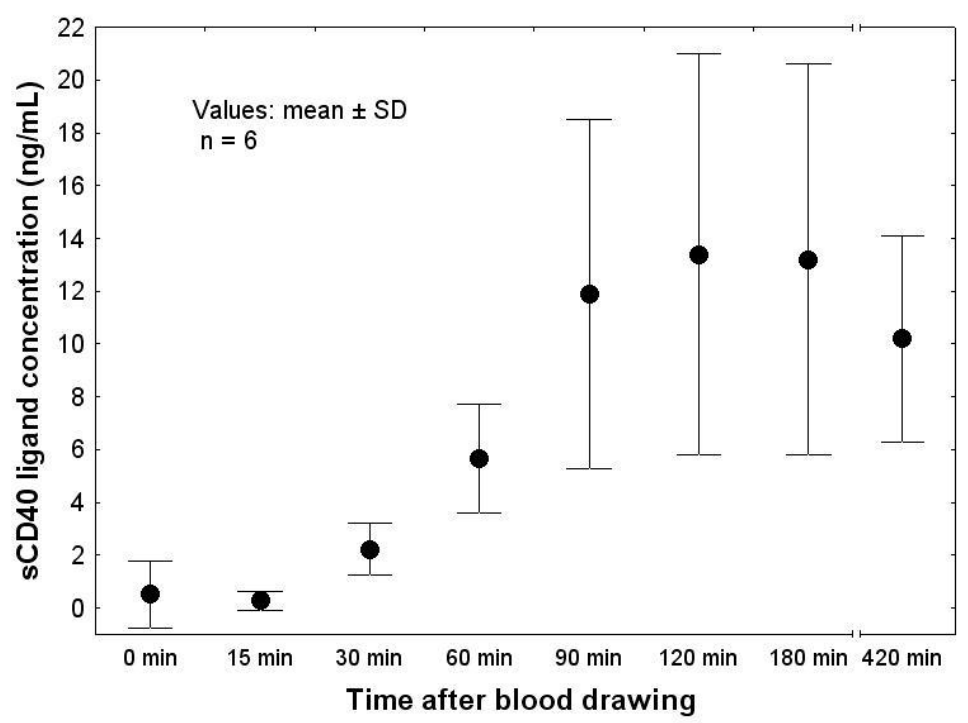

Fig. 1. Serum levels of sCD40L at different time points up till 7 hours after blood sampling in 6 healthy subjects.

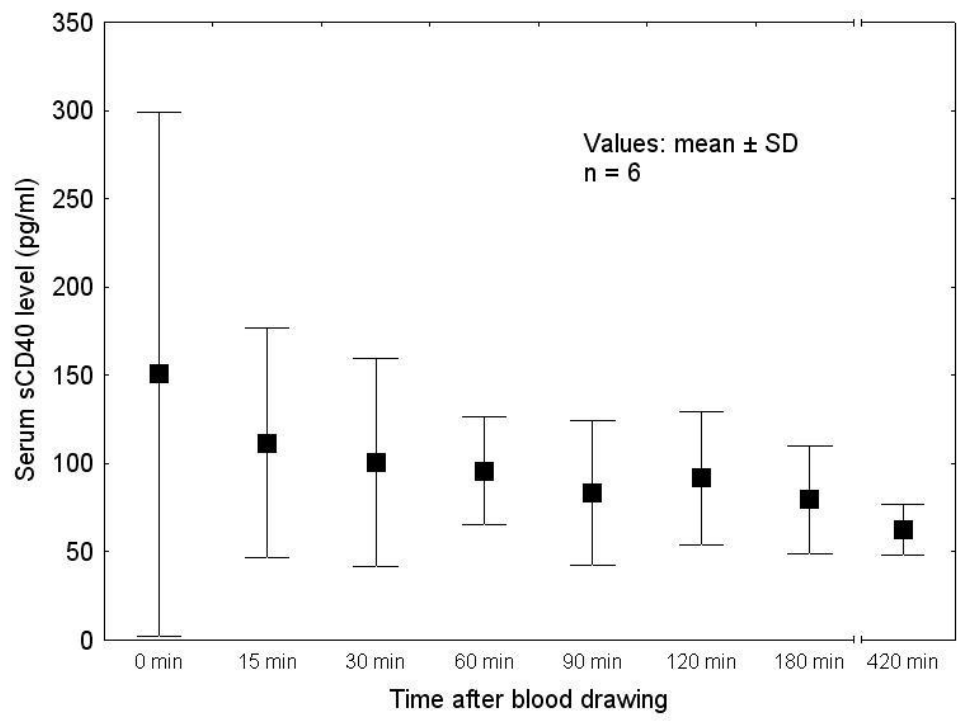

Fig. 2. Serum levels of sCD40 at different time points up till 7 hours after blood sampling in 6 healthy subjects.

lease increased until 90 minutes and reached a plateau between 90 and 120 minutes (Fig. 1). In contrast to SCD40L the sCD40 concentration decreased with the time until 30 minutes and values were stable between 30 and 120 minutes (Fig. 2).

\subsection{Comparison of laboratory parameters and common carotid artery IMT between the groups}

No difference was found in platelet, in red blood cell (RBC) count, in hematocrit and in lipid param- eters including lipoprotein(a) levels between patients and controls. Levels of inflammatory markers as white blood cell (WBC) count $(p=0.0002)$, fibrinogen ( $p=$ $0.0002)$ and $\operatorname{CRP}(p=0.0154)$ were higher in patients with occlusive carotid artery disease. Plasma homocysteine levels were also higher in patients than in controls $(p=0.021)$ (Table 2).

Serum levels of sCD40L were significantly higher $(6.9 \pm 5$ vs. $4.5 \pm 3.0 \mathrm{ng} / \mathrm{mL}, p=0.038)$ in patients, whereas serum levels of sCD40 did not differ significantly between patients and controls $(85 \pm 56.9$ vs. 
Table 2

Blood/serum parameters and common carotid artery intima-media thickness (CCA-IMT) of study participants

\begin{tabular}{lccl}
\hline Feature & Controls & Patients & \multicolumn{1}{c}{$\mathrm{p}$} \\
\hline WBC $(\mathrm{G} / \mathrm{L})$ & $6.1 \pm 1.0$ & $7.5 \pm 1.8$ & 0.0002 \\
RBC (T/L) & $4.7 \pm 0.4$ & $4.7 \pm 0.4$ & 0.551 \\
HTC & $0.43 \pm 0.03$ & $0.44 \pm 0.04$ & 0.094 \\
Platelet (G/L) & $249.4 \pm 57.9$ & $258.4 \pm 71.7$ & 0.638 \\
Fibrinogen (g/L) & $3.1 \pm 0.5$ & $3.7 \pm 0.9$ & 0.0002 \\
CRP (mg/L) & $2.5 \pm 1.9$ & $7.7 \pm 20.7$ & 0.0154 \\
Homocysteine (umol/L) & $11.8 \pm 4.9$ & $13.9 \pm 5.0$ & 0.021 \\
Triglycerides (mmol/L) & $1.7 \pm 0.9$ & $2.0 \pm 1.5$ & 0.706 \\
Cholesterol (mmol/L) & $5.5 \pm 0.9$ & $5.7 \pm 1.1$ & 0.3 \\
HDL-C (mmol/L) & $1.4 \pm 0.4$ & $1.3 \pm 0.4$ & 0.135 \\
LDL-C (mmol/L) & $3.3 \pm 0.9$ & $3.5 \pm 1.0$ & 0.31 \\
Apo-A1 (g/L) & $1.6 \pm 0.3$ & $1.5 \pm 0.3$ & 0.24 \\
Apo-B (g/L) & $1.1 \pm 0.2$ & $1.2 \pm 0.3$ & 0.296 \\
Lp (a) (mg/L) & $251 \pm 259$ & $385 \pm 522$ & 0.437 \\
sCD40 ligand (ng/mL) & $4.5 \pm 3.0$ & $6.9 \pm 5.0$ & 0.038 \\
sCD40 (pg/mL) & $79.3 \pm 18.7$ & $85.0 \pm 56.9$ & 0.344 \\
Offline mean IMT (mm) & $0.78 \pm 0.12$ & $0.89 \pm 0.14$ & 0.0003 \\
read blinded to patient & & & \\
characteristics & & & \\
\hline
\end{tabular}

Values are mean \pm SD. P denotes statistical significance of MannWhitney U test for the comparisons.

Table 3

Univariate Spearman correlations of sCD40L, sCD40 and IMT

\begin{tabular}{lccc}
\hline Possible predictor & SCD40L & sCD40 & Offline measured CCA IMT \\
\hline Age & $0.06(p=0.54)$ & $0.08(p=0.41)$ & $0.028(p=0.78)$ \\
WBC & $0.14(p=0.17)$ & $0.002(p=0.98)$ & $0.3(p=0.004)$ \\
Platelets & $0.29(p=0.005)$ & $-0.0009(p=0.99)$ & $-0.006(p=0.95)$ \\
Cholesterol & $0.05(p=0.603)$ & $0.25(p=0.015)$ & $0.07(p=0.52)$ \\
HDL-C & $-0.05(p=0.64)$ & $0.024(p=0.817)$ & $-0.15(p=0.16)$ \\
LDL-C & $0.02(p=0.87)$ & $0.23(p=0.036)$ & $0.09(p=0.42)$ \\
Lp(a) & $-0.18(p=0.075)$ & $-0.05(p=0.63)$ & $0.002(p=0.98)$ \\
CRP & $-0.009(p=0.92)$ & $-0.08(p=0.448)$ & $0.11(p=0.3)$ \\
Fibrinogen & $-0.051(p=0.63)$ & $-0.068(p=0.528)$ & $0.21(p=0.051)$ \\
Homocysteine & $0.07(p=0.54)$ & $-0.004(p=972)$ & $0.22(p=0.03)$ \\
SCD40L & - & $0.04(p=0.66)$ & $-0.03(p=0.77)$ \\
sCD40 & $0.05(p=0.66)$ & - & $0.109(p=0.308)$ \\
Offline IMT & $-0.03(p=0.77)$ & $0.109(p=0.308)$ & - \\
\hline
\end{tabular}

Values are Spearman $\mathrm{R}$ values, and their statistical significance in parentheses.

$79.3 \pm 18.7 \mathrm{pg} / \mathrm{mL}, p=0.34)$. As an early marker of atherosclerosis, common carotid artery IMT was significantly thicker in patients with occlusive carotid artery disease than in controls ( $p=0.0003)$ (Table 2).

Because more patients than controls were smokers and hypertensives, and more patients than controls had ischemic stroke or TIA in the case history further analyses were done. In the whole study group no difference was found in SCD40L levels and in IMT between genders, between smokers and nonsmokers, between hypertensive and normotensive subjects, between subjects with stroke and subjects without stroke in their case history, between subjects with and without antiplatelet treatment (data not shown).

\subsection{Correlates of SCD4OL values}

In the next step of the analysis all subjects were pooled and we searched for correlates of SCD40L and IMT values in two steps. First, univariate Spearman correlation coefficients were calculated between possible predictors and the sCD40L and sCD40 values (Table 3). In the next step we performed a multivariate analysis for sCD40L values using a general linear model (general regression model) where actually measured WBC, platelet, fibrinogen, CRP and homocysteine values were used as continuous predictors, and smoking status, a factor known to have an effect on atherosclerosis, as categorical factor (Table 4). From 
Table 4

Results of multivariate analysis (GLM, general regression model) on the effects of possible predictors of sCD40L

\begin{tabular}{lcc}
\hline Possible predictor of sCD40L & $\beta$ and 95\% CI & $\mathrm{p}$ \\
\hline Platelet count & 0.357 & 0.014 \\
WBC & $(0.075-0.64)$ & \\
& -0.053 & 0.697 \\
CRP & $(-0.33-0.22)$ & \\
& 0.209 & 0.169 \\
Fibrinogen & $(-0.092-0.511)$ & \\
Homocysteine & -0.404 & 0.012 \\
& $(-0.718--0.09)$ & \\
Smoking & 0.124 & 0.325 \\
& $(-0.127-0.375)$ & \\
& $(-0.508--0.011)$ & \\
\hline
\end{tabular}

$\beta$ : regression coefficient; CI: confidence interval; GLM: general linear model. For the whole model the key variables are as follows: multiple $R=0.49$, multiple $R^{2}=0.24, p=0.022$.

the univariate analysis it can be seen that without controlling for other factors, platelet count had significant association with sCD40L values (Table 3). Although sCD40L levels correlated with platelet counts $(R=$ $0.29, p=0.005)$, in multivariate testing the difference in sCD40L levels between patients and controls remained significant $(p=0.003)$ after controlling for the effect of platelet count, WBC, CRP and fibrinogen levels. General regression analysis revealed that platelet count and fibrinogen had strong effects on sCD40L, even after controlling for WBC, CRP, homocysteine and smoking status (Table 4).

\subsection{Correlates of CCA IMT values}

Actually measured white blood cell (WBC) count and homocysteine level significantly associated with common carotid artery IMT values (Table 3 ). In multivariate testing WBC count remained in significant association with IMT (multiple $R=0.35$, multiple $R^{2}=$ $0.125, p=0.003$ ) even after controlling for the effect of the categorical predictors (gender, hypertensive status, stroke in case history) which were on the border of statistical significance in univariate Mann-Whitney testing (multiple $R=0.44$, multiple $\mathrm{R} 2=0.19, p=0.041$ ). CCA IMT, an early marker of atherosclerosis, did not correlate with sCD40L or sCD40 levels $(R=-0.03$, $p=0.77$; and $R=0.109, p=0.308$, respectively).

\section{Discussion}

\subsection{CD40-CD40L system and inflammation/atherosclerosis}

In recent years the role of inflammation in atherosclerosis has been emphasized [25]. Activation of the
CD40-CD40L axis was shown to occur in chronic inflammatory diseases such as autoimmune disorders [26], in atherosclerosis [27] or in cancer [28]. Another study has supported the role of CD40-CD40L interactions in atherosclerosis, thrombosis and inflammation [29]. The CD40-CD40L axis upregulates proinflammatory mediators, such as the expression of adhesion molecules (ICAM-1, VCAM-1) and synthesis of proinflammatory cytokines (TNF- $\alpha$, IL-6), enhances the production of matrix-metalloproteinases and chemokines (MCP-1) [2]. All of these mediators induce a potent inflammatory reaction of the vascular endothelial cells to provoke atherosclerosis [6].

\subsection{Platelets and inflammation/atherosclerosis}

Platelets are important factors of inflammation as well as hemostasis. They possess adhesion molecules, which facilitate interactions with neutrophils, monocytes and endothelial cells, and can activate these cells through direct contact or through the release of soluble mediators. Platelets express the transmembrane signaling protein CD40L within seconds of activation, and after platelets activation, a soluble form of CD40L is also released [6]. Both membrane-bound and SCD40L may have biological effect on a number of cell types [6] and exhibit procoagulant activity [7]. Furthermore, sCD40L binding to platelet CD40 leads to further cleavage of membrane-bound CD40L and release of sCD40L [6]. These suggest an important pathophysiological role of platelets in triggering not only thrombosis, but also vascular inflammation.

In our study we measured maximal platelet-derived sCD40L levels. Initially, we tried to use plasma samples for the sCD40L measurements. However, as suggested by the manufacturer this could not be done reliably [30], and we could not measure SCD40L in either frozen or fresh plasma samples [31]. This might have two reasons. First, the method itself is not suitable for plasma samples [30]. Second, there is indeed no measurable baseline levels of sCD40L in plasma, and whatever $\mathrm{sCD} 40 \mathrm{~L}$ concentration is measured, it is released to the plasma or serum after platelet activation. Semb et al. [32] reported that SCD40L was nearly undetectable in healthy controls, and even those studies that reported some measurable amount, there was a tenfold difference in control values between studies $(0.37$ $\pm 0.21 \mathrm{ng} / \mathrm{mL}-3.4 \pm 1.6 \mathrm{ng} / \mathrm{mL},[10,14])$. To check for a possible reason of these differences we measured the time-dependent release of CD40L and CD40 on 6 healthy subjects. Sampling methods are known to af- 
fect the results of sCD40L assays [33]. Although most probably sCD40L values measured from platelet-free plasma samples approximate best the in vivo levels of sCD40L, it can not be excluded that the procedure of blood sampling itself activates platelets thus results in higher sCD40L levels even in these samples compared to the circulating in vivo levels. Because of this uncertainty and as we could not measure any sCD40L level in the plasma samples, we decided that instead of trying to evaluate in vivo circulating levels of SCD40L, we repeat the study and measure the total amount of releasable sCD40L. Therefore we allowed non-citrated blood samples to clot for 90-120 minutes, and serum samples were used for sCD40L assay.

We found significantly elevated sCD40L levels in patients with early onset occlusive carotid artery disease. Although sCD40L levels correlated with platelet count, this difference in SCD40L levels between patients and controls remained significant after controlling for platelet count. In recent studies increased levels of sCD40L were observed in acute coronary syndromes [9], acute cerebral ischemia [10] and peripheral arterial occlusive disease [11]. To our knowledge, this is the first time to examine sCD40L levels in patients with early onset of carotid atherosclerosis. We found that sCD40L significantly correlated with platelet count and fibrinogen, after controlling for other factors (Table 4). Since platelet count did not differ significantly between the patients and controls ( $p=$ 0.638) (Table 2) and as the SCD40L difference between patients and controls remained significant after controlling for platelet count, it seems that patients with occlusive carotid artery disease have a chronic inflammation state with elevated inflammatory markers, and activated platelets may release larger amounts of sCD40L. Although we did not measure platelet activation directly, as most of the sCD40L is derived from platelets [8], our findings suggest that chronic platelet activation and platelet derived SCD40L may be the linkage between inflammation and the early onset of atherosclerosis.

\section{Acknowledgements}

This study was supported by grants from the Health Science Council of the Ministry of Health of the Hungarian Republic (ETT-130/2003-041/037), from DEOEC MEC 08/2005, University of Debrecen and from the Hungarian Research Foundation (OTKA037883). Costs of the sCD40 and sCD40L ELISA kits were covered by an unrestricted grant from SanofiSynthélabo Hungary. We thank Virág Jánosné and Molnár Andrásné for technical assistance.

\section{References}

[1] D. Graf, G.D. Korthauer, H.W. Mages, G. Segner and R.A. Kroczek, Cloning of TRAP, a ligand for CD40 on human T cells, Eur J Immunol 22 (1992), 3191-3194.

[2] U. Schonbeck, F. Mach and P. Libby, CD154 (CD40 ligand), IJBCB 32 (2000), 687-693.

[3] K. Buchner, V. Henn, M. Grafe, O.J. de Boer, A.E. Becker and R.A. Kroczek, CD40 ligand is selectively expressed on CD4+ T cells and platelets: implications for CD40-CD40L signalling in atherosclerosis, J Pathol 201 (2003), 288-295.

[4] D.P. Inwald, A. McDowall, M.J. Peters, R.E. Callard and N.J. Klein, CD40 is constitutively expressed on platelets and provides a novel mechanism for platelet activation, Circ Res 92 (2003), 1041-1048.

[5] F. Mach, U. Schonbeck, G.K. Sukhova, E. Atkinson and P. Libby, Reduction of atherosclerosis in mice by inhibition of CD40 signalling, Nature 394 (1998), 200-203.

[6] V. Henn, J.R. Slupsky, M. Grafe, I. Anagnostopoulos, R. Forster, G. Muller-Berghaus and R.A. Kroczek, CD40 ligand on activated platelets triggers an inflammatory reaction of endothelial cells, Nature 391 (1998), 591-594.

[7] F. Mach, U. Schonbeck, J.Y. Bonnefoy, J.S. Pober and P. Libby, Activation of monocyte/macrophag functions related to acute atheroma complication by ligation of CD40: induction of collagenase, stromelysin, and tissue factor, Circulation 96 (1997), 396-399.

[8] M. Nagasawa, Y. Zhu, T. Isoda, D. Tomizawa, S. Itoh, M. Kajiwara, T. Morio, S. Nonoyama, N. Shimizu and S. Mizutani, Analysis of serum soluble CD40 ligand (sCD40L) in the patients undergoing allogeneic stem cell transplantation: platelet is a major source of serum sCD40L, Eur J Haematol 74 (2005), 54-60.

[9] P. Aukrust, F. Muller, T. Ueland, T. Berget, E. Aaser, A. Brunsvig, N.O. Solum, K. Forfang, S.S. Froland and L. Gullestad, Enhanced levels of soluble and membrane bound CD40 ligand in patients with unstable angina, Circulation $\mathbf{1 0 0}$ (1999), 614-620.

[10] C.D. Garlichs, S. Kozina, S. Fateh-Moghadam, B. Tomandl, C. Stumpf, S. Eskafi, D. Raaz, A. Schmeiser, A. Yilmaz, J. Ludwig, B. Neundörfer and W.G. Daniel, Upregulation of CD40-CD40 ligand (CD154) in patients with acute cerebral ischemia, Stroke 34 (2003), 1412-1418.

[11] D.A. Tsakiris, M. Tschopl, F. Wolf, K.H. Labs, K.A. Jager and G.A. Marbet, Platelets and cytokines in concern with endothelial activation in patients with peripheral arterial occlusive disease, Blood Coagul Fibrinolysis 11 (2000), 165-173.

[12] N. Varo, D. Vicent, P. Libby, R. Nuzzo, A.L. Calle-Pascual, M.R. Bernal, A. Fernandez-Cruz, A. Veves, P. Jarolim, J.J. Varo, A. Goldfine, E. Horton and U. Schonbeck, Elevated plasma levels of the atherogenic mediator soluble CD40 ligand in diabetic patients: a novel target of thiazolidimediones, Circulation 107 (2003), 2664-2669.

[13] C.D. Garlichs, S. John, A. Schmeisser, S. kafi, C. Stumpf, M. Karl, M. Goppelt-Struebe, R. Schmieder, W.G. Daniel and A. Schmeiser, Upregulation of CD40 and CD40 ligand (CD154) in patients with moderate hypercholesterolemia, Circulation 104 (2001), 2395-2400.

[14] S.A. Harding, J. Sarma, D.H. Josephs, N.L. Cruden, J.N. Din, P.J. Twomey, K.A.A. Fox and D.E. Newby, Upregulation of the CD40/CD40 ligand dyad and platelet-monocyte aggregation in cigarette smokers, Circulation 109 (2004), 1926-1929. 
[15] U. Schonbeck, N. Varo, P. Libby, J. Buring and P.M. Pidker, Soluble CD40L and cardiovascular risk in women, Circulation 104 (2001), 2266-2268.

[16] M.T. Magyar, Z. Szikszai, J. Balla, A. Valikovics, J. Kappelmayer, S. Imre, G. Balla, V. Jeney, L. Csiba and D. Bereczki, Early onset carotid atherosclerosis is associated with increased intima-media thickness and elevated serum levels of inflammatory markers, Stroke 34 (2003), 58-63.

[17] M. Zimarino, L. Cappelletti, V. Venarucci, S. Gallina, M. Scarpignato, N. Acciai, A.M. Calafiore, A. Barsotti and R. De Caterina, Age-dependence of risk factors for carotid stenosis: an observational study among candidates for coronary arteriography, Atherosclerosis 159 (2001), 165-173.

[18] M.R. de Groot and J.D. Banga, Non invasive ultrasound measurement of intima-media thickness. A tool to measure early atherosclerosis, Eur J Vasc Surg 8 (1994), 257-263.

[19] A. Simon, J. Gariepy, G. Chironi, J.L. Megnien and J. Levenson, Intima-media thickness: a new tool for diagnosis and treatment of cardiovascular risk, J Hypertens 20 (2002), 159169.

[20] D. Baldassarre, M. Amato, A. Bondioli, C.R. Sirtori and E. Tremoli, Carotid artery intima-media thickness measured by ultrasonography in normal clinical practice correlates well with atherosclerosis risk factors, Stroke 31 (2000), 2426-2430.

[21] K. Winbeck, C. Kukla, H. Poppert, J. Klingenhofer, B. Conbrad and D. Sander, Elevated C-reactive protein is associated with an increased intima to media thickness of the common carotid artery, Cerebrovasc Dis 13 (2002), 57-63.

[22] European Carotid Surgery Trialists' Collaborative Group, MRC European carotid surgery trial: Interim results for symptomatic patients with severe (70-99\%) stenosis or with mild (0-29\%) stenosis, Lancet 337 (1991), 1235-1243.

[23] B. Widder, G.M. v Reutern and D. Neuerburg-Heusler, Morphologische und dopplersonographische Kriterien zur Bestimmung von Stenosierunsgraden an der A. carotis interna, $U l$ traschall Med 7 (1986), 70-75.
[24] G. Howard, A.R. Sharrett, G. Heiss, G.W. Evans, L.E. Chambless, W.A. Riley and G.L. Burke, Carotid artery intimal-medial thickness distribution in general populations as evaluated by B-mode ultrasound, Stroke 24 (1993), 1297-1304.

[25] R. Ross, Atherosclerosis-an inflammatory disease, N Engl J Med 340 (1999), 115-126.

[26] S.K. Datta and S.L. Kalled, CD40-CD40 ligand interaction in autoimmune disease, Arthritis Rheum 40 (1997), 1735-1745.

[27] J.D. Laman, B.J. de Smet, A. Schoneveld and M. van Meurs, CD40-CD40L interactions in atherosclerosis, Immunol Today 18 (1997), 272-277.

[28] P.W. Cooke, N.D. James, R. Ganesan, M. Wallace, A. Burton and L.S. Young, CD40 expression in bladder cancer, $J$ Pathol 188 (1999), 38-43.

[29] U. Schonbeck and P. Libby, CD40 signaling and plaque instability, Circ Res 89 (2001), 1092-1103.

[30] Bender MedSystems, Human sCD40L Instant ELISA. Product information and manual. Vienna, Austria: MedSystems Diagnostics GmbH; 2001:23.

[31] D. Bereczki, E. Nagy, A. Pál, M.T. Magyar and J. Balla, Should soluble CD40 ligand be measured from serum or plasma samples? Arterioscler Thromb Vasc Biol 23 (2003), 1129-1130.

[32] A.G. Semb, S. van Wissen, T. Ueland, T. Smilde, T. Waehre, M.D. Tripp, S.S. Froland, J.J. Kastelein, L. Gullestad, T.R. Pedersen, P. Aukrust and A.F. Stalenhoef, Raised serum levels of soluble CD40 ligand in patients with familial hypercholesterolemia: downregulatory effect of statin therapy, J Am Coll Cardiol 41 (2003), 275-279.

[33] E.R. Ahn, G. Lander, W. Jy, C.J. Bidot, J.J. Jimenez, L.L. Horstman, and Y.S. Ahn, Differences of soluble CD40L in sera and plasma: implications on CD40L assay as a marker of thrombotic risk, Thromb Res 114 (2004), 143-148. 


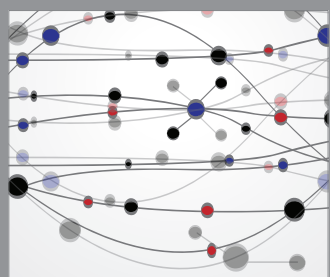

The Scientific World Journal
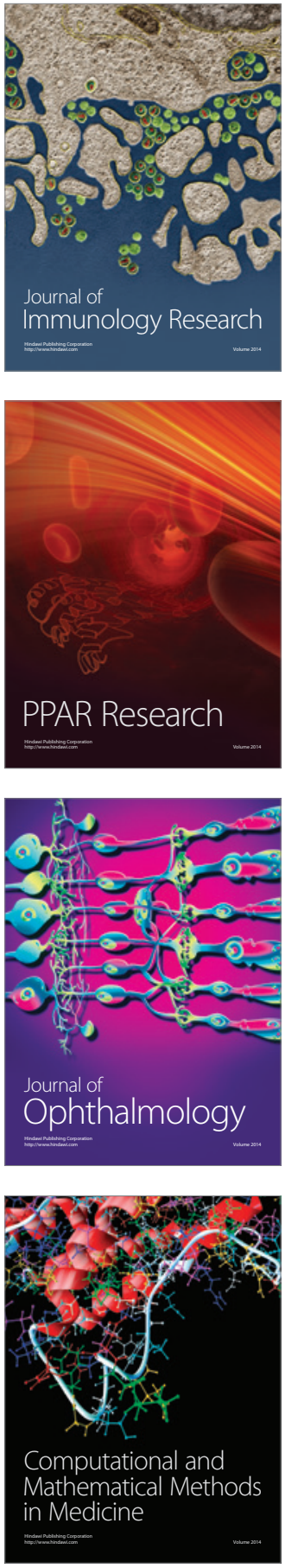

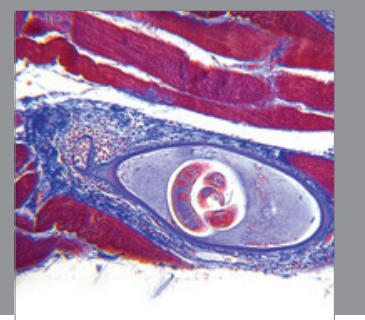

Gastroenterology

Research and Practice
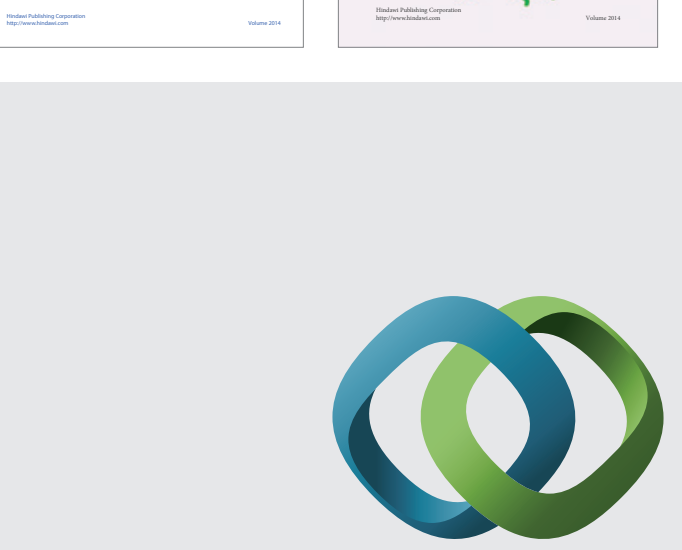

\section{Hindawi}

Submit your manuscripts at

http://www.hindawi.com
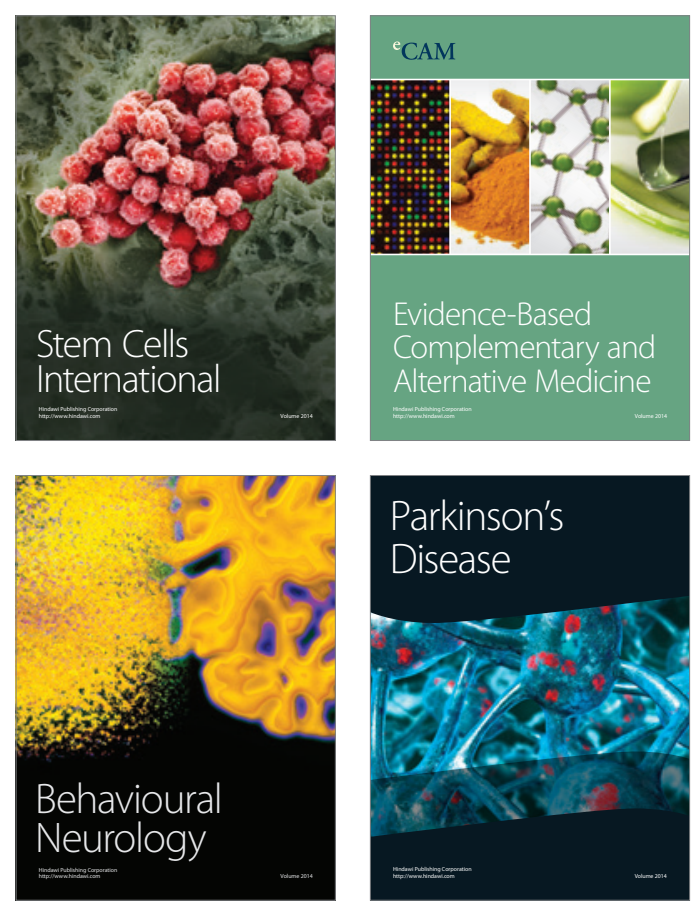

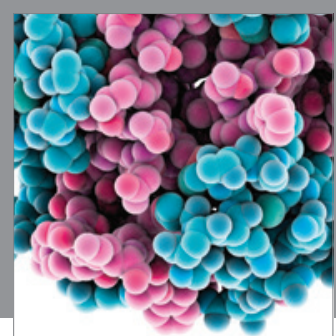

Journal of
Diabetes Research

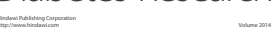

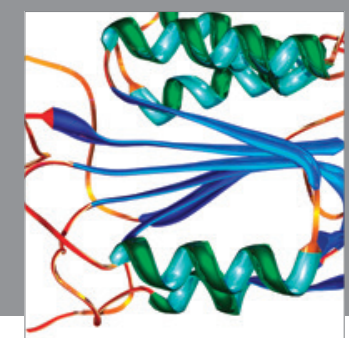

Disease Markers
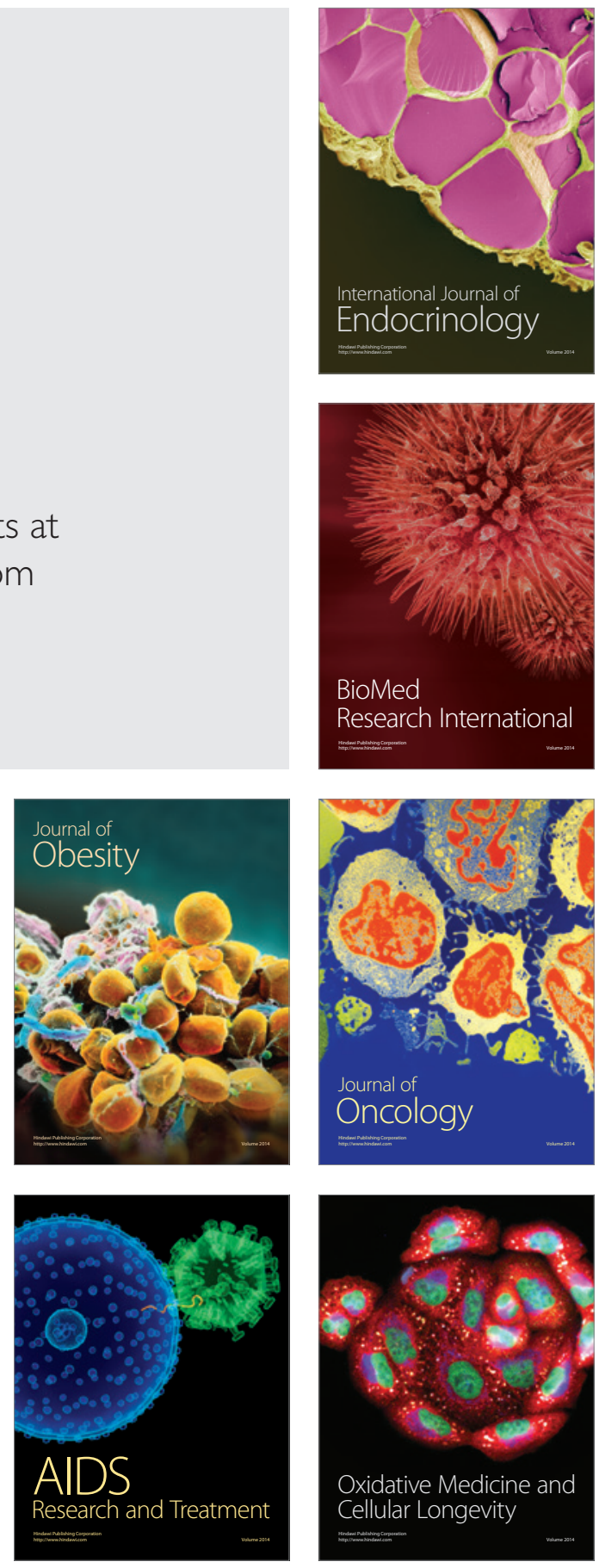